\title{
A Requisite of a Permittivity of the Interfacing Medium for the Electromagnetic Surface Excitation by a Vertical Electric Dipole nearby the Interface
}

\author{
Yoshinari ISHIDO
}

\author{
Photonic Device Application Group, Research Institute for Ubiquitous Energy Devices, AIST Kansai
}

Received March 20, 2014, Accepted October 30, 2014

\begin{abstract}
In planer-type optical devices such as OLED, the loss-propagating surface plasmon polaritons (SPPs) at the interface on a metallic cathode has been regarded as holding the key to the efficiency improvement. It complicates the characteristics of SPPs, however, that the existence of the classical or phenomenological surface electromagnetic (EM) propagation modes at the interface. With the aid of tracing the predecessors' prior achievements, we clarify theoretically the characteristic of surface EM modes over a comprehensive variety of a permittivity of the interfacing medium. As a result, we prove that there is a requisite in order that the surface EM excitations might be quasi independently observed in a vertical dipole radiation nearby the interface, which is non-positive real part of a permittivity of the medium interfacing to the air, such as a real metal, involving the condition for existence of SPPs.
\end{abstract}

KEYWORDS: Organic Light Emitting Diode, dipole nearby a plane interface, surface wave excitation, surface plasmon loss, Zenneck mode

\section{Introduction}

Improvements of light source extraction or out-coupling in organic light emitting diodes (OLED's) are important ${ }^{1)}$. One of the dominant of barriers to them has been said to be a loss at the surface of the metallic cathodes of OLED, which is considered to be caused by the surface EM excitations at the interface between the metallic cathode and the fluorescent thin film layer, so called "surface plasmon loss" from quasi particle viewpoint ${ }^{2}$. For evaluating the light extraction or the losses in OLED, an electric dipole near the interface of media is often used as a fluorescent model for calculation ${ }^{233}$, whose theoretical background is rather classical, as seen in the followings.

Zenneck \& Sommerfeld treated electromagnetic (EM) surface excitations at, or propagation along the "earth ground-air" interface in radio frequency ranges or the "flat metal-air" in optical frequency ranges by a vertical electric dipole antenna near there, in 1907 \& 09, respectively ${ }^{45)}$. Since then, a great deal of researches, formerly theoretical in main, latterly experimental on various scopes has done $e^{6 / 7}$.

In classic \& phenomenological electromagnetism, it has a right to propagate along the interface of two media that only the one p-polarized surface wave mode, which has alternative phases as the difference of interfacing media condition ${ }^{7}$ : First, in the case of one medium with a high conductivity, the mode is often called the "Zenneck mode" which is a fast mode with conductive losses. Second, at the interface of two non-absorbing media whose permittivities have opposite signs to each other, a lossless slow-guided mode must exist, which is called the "Fano mode". Last, as the limit case that the interface consists of a pair of lossless media both of which have positive real permittivities, its EM fields are no binding to the interface however, it has a lossless propagating constant corresponding to a Brewster angle, and so, sometime called "Brewster angle EM mode". It is most remarked that these modes represent the same singularity defined by the same equation socalled characteristic equation derived from pairs of EM wave on each side to the interface of two media which should fulfill the boundary conditions by themselves in all the phases.

Nevertheless above mentions, the observation of a surface EM mode excitation by an electric dipole near the interface has not been sufficiently realized, except on such an experimental viewpoint as the excitation should be concerned with the surface plasmon polaritons (SPPs) for a real metal having a negative permittivity in visible spectral ranges ${ }^{8}$.

The SPPs are regarded as the quantum states referred to plasma oscillations in the surface excitation modes. At the interface between a metal and a lossless dielectric, the SPPs have the component of electromag- 
netic waves, and are quantized as the SPPs which can interact "slow-photons" in the vicinity of the plasma frequency $^{9)}$. It is most remarked that the attribution of a "polariton" in the SPPs corresponds to the classical and phenomenological surface EM wave modes at the interface, which is away from that of a "plasmon" in SPPs ${ }^{10}$. For example, the permittivity of an interfacing medium as $\varepsilon<-1$, which is often considered the requirement for existence of SPPs ${ }^{11}$, is substantially the condition that the surface wave (SW) mode corresponding to the polaritons in SPPs should become lossless, that is Fano mode already mentioned.

Then it appears why the SPPs have been observed chiefly on the surface of metals, nevertheless the SW modes including loss ones can exist in all varieties of a permittivity of the interfacing media. Thus, a self-consistent description of a surface EM mode excitation from the viewpoint of EM theory for continuous media has been obliged to be established still more.

According to a great deal of prior theoretical mentions the residue of the pole corresponding to the SW mode has been always cancelled by a piece of the amounts of the integral represented by a superposition of radiative plane fields emerging from all complex angles, which means that the SW mode is a loss mode, degenerated by other radiative modes, therefore hidden by a non-geometric optical component of the ground wav, so-called "Norton surface wave ${ }^{6) 12}$.

Thus, it should be clear that do we it is to deduce at least an indispensable condition of embodying a surface EM excitation with respect to a permittivity of the interfaced media from the viewpoint of the phenomenological EM wave theory, using the prior theoretical results and achievements as bases.

Additionally, the square root as a multi valued complex function denotes $(\cdot)^{1 / 2}$, instead of $\sqrt{ }$, consistently in this article.

\section{Norton's attenuation factor as a complex function}

In his 1937's literature (12), Norton proposed the expression of radiative EM fields from an electric dipole nearby the "ground-air" interface suitable for analyzing propagation over the ground, based on the radiation

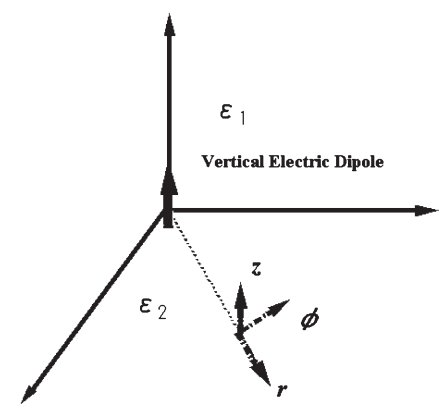

Figure 1 Vertical electric dipole at the interface formula which has been formerly divided by Sommerfeld into a "space" component and a "surface" component; the latter in other words, a "lateral" component to distinguish from the SW mode above mentioned ${ }^{6}$.

The geometry in Norton's notation is drawn as Figure 1 , which is a special case that the vertical electric dipole is grounded at the interface.

As the "space" component eliminates when the observation point is near the ground, it only remains that the "lateral" component, whose electric fields at the extremely high frequency are as follows:

$$
\begin{aligned}
E_{\text {lateral }}= & -2 \mathrm{j} F \exp \{\mathrm{j}(\omega t-k R)\} / R \\
& \times\left[\mathrm{k}-\mathbf{r}\left(1+z^{2} / 2 R^{2}\right) u\left(1-u^{2}\right)^{1 / 2}\right]
\end{aligned}
$$

$R$ is the distance from the ground source to the observation point whose height from the surface is denoted by $z \cdot u$ is equal to $1 /\left(\varepsilon_{2}\right)^{1 / 2} ; \varepsilon_{2}$ is a complex permittivity of the medium interfacing to the air, and:

$$
F=1-\mathrm{j}(\pi \rho)^{1 / 2} \operatorname{erfc}\left(\mathrm{j} \rho \rho^{1 / 2}\right)
$$

Equation (2) is so-called Norton's attenuation factor for his precise description about. And $\rho$ is called a numerical distance, with Norton's notation, as;

$$
\begin{aligned}
\rho & =-\mathrm{j}(1 / 2) k R\left[u\left(1-u^{2}\right)^{1 / 2}-(z / R)\right]^{2} \\
& \equiv \phi \exp [-\mathrm{j} b]
\end{aligned}
$$

Norton and other researchers have displayed the dependences of a magnitude and an argument of the factor respectively upon a numerical distance; clarified inherent attenuations, unlike of surface waves. The factor $F$ however, as it is a complex function in general, has a real part and an imaginary both of which should bear respective physical meanings: it is equivalently re-derived from Equation (2), with the use of general formulae on the error functions, as follows ${ }^{12-15)}$.

$$
F=1-\mathrm{j}(\pi \rho)^{1 / 2} e^{-\rho}-2 \rho^{1 / 2} e^{-\rho} \int_{0}^{(\rho)^{1 / 2}} \exp \left(\mathcal{\lambda}^{2}\right) \mathrm{d} \mathcal{\lambda}
$$

Sommerfeld pointed out, in his latter tutorial literature ${ }^{15}$, the second term of Equation (4) should correspond to the Zenneck's SW which has a propagation constant $\lambda_{\mathrm{p}}$ with $\rho \sim-\mathrm{j}\left(k-\lambda_{\mathrm{p}}\right) r$, as $z \rightarrow 0$;

$$
(\pi \rho)^{1 / 2} e^{-\rho} \exp (-\mathrm{j} k R) / R \propto \exp \left(-\mathrm{j} \lambda_{\mathrm{p}} r\right) / r^{1 / 2}
$$

which vanishes more gradually than a far field of radiation except the exponential loss by the imaginary part of $\lambda_{\mathrm{p}}$.

On the other hand, as the second term has a complex unit explicitly, it is only the one which should value pure imaginary, separable from the other "radiative" terms in the "lateral" component, if $\rho$ is a pure real.

Let us re-draw the attenuation curve of the factor $F$, which is wholly complex-valued, as seen in Figure 2.

Note that the magnitude of an imaginary part of the attenuation factor $F$ has a maximum value with respect to $p$, for all $b$. Moreover its amount becomes optimum at 

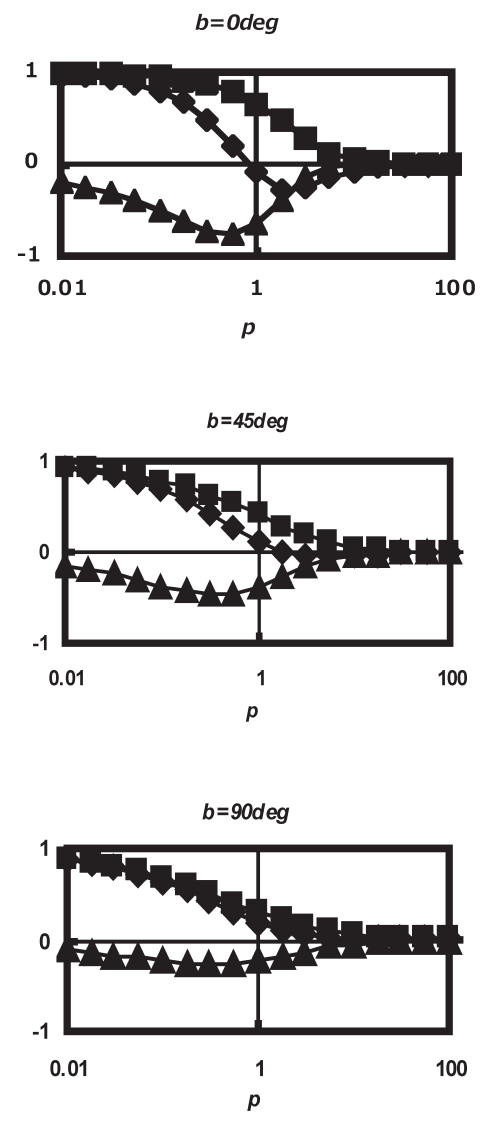

Figure 2 Norton's attenuation factors versus a numerical distance: $\boldsymbol{\square}=$ =absolute; $\boldsymbol{\nabla}=$ real part; $\mathbf{\Delta}=$ =imaginary part, respectively.

$b=0$ degree, which is valued in the vicinity of the zero point of a real part of $F$ with respect to $p$, when $p$ is about unit.

Additionally it means that the field is dominated wholly by the second term of $F$ in this case, $b=0$ degree and $p$ is about unit, where the field is shaped to be nonradiative. Otherwise, the contribution of the second term has been degenerated to the pieces of both real and imaginary part of the amounts of $F$, and so the SW's properties also are hidden in those of the "lateral" component ${ }^{6)}$.

This result induces us to investigating a condition or a requisite that the numerical distance $\rho$ should become positive real, concerning with a variety of the interfacing media especially: We should prepare another theoretical tool which is called a steepest descent method for reducing the branch integral representation in the general formula as seen in Appendix.

3. Complex analysis of the SW excitation at the lossy interface using a steepest descent method

From a definition of $T$ as A(18) in Appendix, we see that its null argument is equivalent to a pure real of the numerical distance $\rho$, as the argument ranges from $-\pi / 4$ to $\pi / 4$.

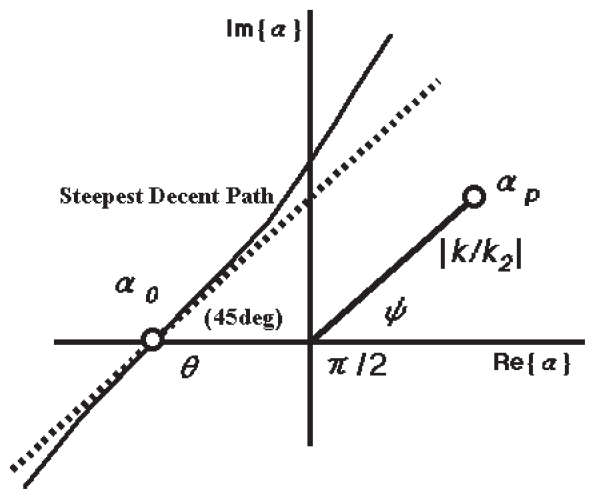

Figure 3 Geometry of the saddle point, $\pi / 2$ point, and the pole

Substituting (A13) and (A16) into (A18), it is derived;

$$
T=\left(a_{p}-a_{0}\right) \exp (-\mathrm{j} \pi / 4)
$$

Then we find that the $\pi / 4$ argument of $\left(a_{p}-a_{0}\right)$ is also equivalent to the pure real of the numerical distance.

Now let us sketch the geometric arrangement of $a_{p}$ and $a_{0}$, with a Steepest Decent Path (SDP) in the $a$ plane, in Figure 3.

In this figure, the dashed line is a tangent of SDP at the saddle point $a_{p}$ with $\pi / 4$ angle from the real axis.

First, we can find that the $\pi / 4$ argument of $\left(a_{p}-a_{0}\right)$ is also equal to the pole $a_{p}$ located on the tangent. Additionally, note that, as $\left|k / k_{2}\right| \ll 1$ and $\theta \ll 1$ respectively, $a_{p}$ is in the neighborhood of $a_{0}$, where the SDP may be regarded as the same as a tangent of itself.

Then, the condition of the numerical distance being a positive real is equivalent to that of the pole being just located on the SDP near the saddle point. And simultaneously, an equation may be revealed by substituting equation (A18) into that condition, as $\sin \theta \sim \tan \theta \sim \theta$ $\ll 1$ :

$$
\tan \theta \sim \operatorname{Im}\left\{k / k_{2}\right\}-\operatorname{Re}\left\{k / k_{2}\right\}
$$

Next, it should be remarked that the location of the pole is dominated by the argument of $k / k_{2}, \phi$, which is a half of the dielectric $\varepsilon_{2}$ loss angle denoted as $\phi$ in equation (A5). It is also noted that $a_{0}$ should be located on the left hand of the real axis. Then, the geometrical relation is drawn in Figure 4 divided into three cases:

Case(a): The pole $a_{p}$ is never located on the SDP. Therefore the numerical distance $\rho$ can never become positive real.

Case(b): It exists that some $\theta_{1}$, namely $R_{1}$ and $z_{1}$ as the pole $a_{p}$ is located on the SDP, so the numerical distance $\rho$ is a positive real.

Case(c): It is only in the limit of $\theta \rightarrow 0$, namely $z=0$ or $R=\infty$ that the pole $a_{p}$ may be located on the SDP, so that the numerical distance $\rho$ be a positive real.

These cases (a), (b), and (c) are corresponding to $\phi<\pi / 2$, or $\operatorname{Re}\left\{\varepsilon_{2}\right\}>0 ; \phi>\pi / 2$, or $\operatorname{Re}\left\{\varepsilon_{2}\right\}<0$; and $\phi=\pi / 2$, or 


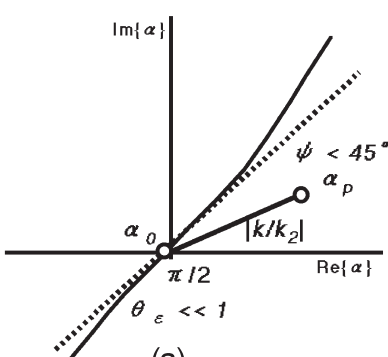

(a)

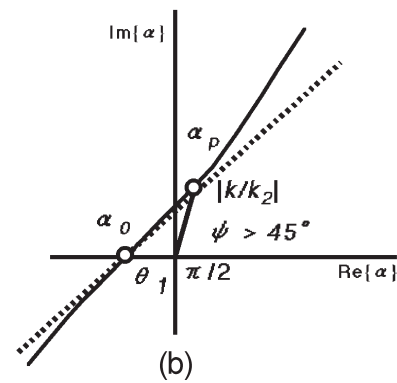

(b)

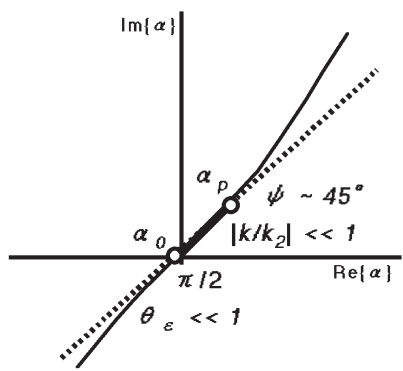

(c)

Figure 4 Three cases of the geometry with respect to $k / k_{2}$

$\operatorname{Re}\left\{\varepsilon_{2}\right\}=0 ; \quad$ respectively.

As a result, the Zenneck type surface wave may be observed independently of the other "radiative-lateral" components on condition that the numerical distance is valued about unit, if and only if a real part of the permittivity of a medium interfacing to the free space is nonpositive, whose both of bounds correspond to the "Zenneck mode" and the "Fano mode", respectively.

Then, we have one question: Is the non-positive real part of that permittivity enough for realizing the surface excitation? As a matter of fact, the above mentions are based on an approximated representation which consists of only a surface wave field under near fields and of far fields. Providing that it is a rigorous representation, the field integral $S$ seen in Appendix has an integral path along the axis denoted as $\operatorname{Im}\{a\}$ in Figure 3. Then, the case that the pole is located on the integral path is equivalent to $\phi=\pi / 2(\phi=\pi)$, where the mode is restricted to a "Fano mode". As already seen, only the "Fano mode" is lossless, independent of the other "radiative" modes in the field of energy, therefore, is a no- "improper" mode, borrowing Wait's words in reference (6), which means an only steady-state mode propagating along the interface of a two-layer lossless system. This fact seems the reason why the lossless "Fano mode" is often regarded as the same as ideal SPPs ${ }^{11}$. It is also remarked that the $\pi / 2-\theta$ is convergent to the Brewster angle, according to Equation (7) on the "Fano mode" ${ }^{21}$.

In additional, returning to Figure (2), we discuss to inspire a physical meaning of the imaginary part of the Norton's attenuation factor.

Let us compare the retardation between the phase of a "lateral" component and that of a "space" component. According to reference (16), the ratio of magnitudes of electric vertical components between "spatial" and "lateral" is;

$$
E_{\text {latetal }}^{k} / E_{\text {space }}^{k} \propto\left\{\left(1-R_{V}\right) /\left(1+R_{V}\right)\right\} F
$$

In the above equation, $R_{V}$ denotes a reflective component. Providing that $R_{V}$ is real, it is found that the "radiative-lateral" term has no retardation to the "space" components. On the contrary, it is also found that the "surface" term has $\pi / 2$ retardation to the "space" component. This $\pi / 2$ retardation between the phase of the "surface" term and that of the "space" component is well- analogous to the same retardation between an electrical tangential component in a surface mode and that in a guiding p-mode of an open slab type waveguide.

\section{Conclusions and remarks}

We show the demonstration of a requisite as a following sentential sequence:

(S1) There is an optimum condition of the imaginary part of the Norton's attenuation factor corresponding to the surface wave, which is just independent of the other radiative waves.

$\Rightarrow$ (S2) The numerical distance should be a positive real.

$\Leftrightarrow$ (S3) In the complex wave number plane, the pole corresponding to the surface wave mode can be located on the SDP of the integral representation of the radiation field.

$\Rightarrow$ (S4) The argument of the pole is over 45 degree; provided that the interfacing medium has a large magnitude of its complex permittivity.

$\Rightarrow$ (S5) Its loss angle is over 90 degree.

$\Leftrightarrow$ (S6) The real part of a permittivity of that medium is non-positive, such as a real metal in visible spectral ranges.

Namely, Sentence 6 (S6) has been clarified to be a requisite of Sentence 1 (S1).

As already mentioned in Introduction, the only media having a negative real part of their permittivity are metals in visible light. Therefore, the SW excitation cannot be disregarded on the electroluminescence with metal-electrodes, for an illumination device.

As the optimum condition of a numerical distance for a "surface" term's dominating, $p \sim 1$, we may estimate a required distance from a dipole to a SW excitation; that is, $r \sim\left|k_{2} / k\right|\{\mathcal{X} /(2 \pi)\}$ as $\phi \sim \pi / 2$ (Zenneck mode), and $r \sim\left(\left|k_{2} / k\right|^{2}-1 / 2\right)(\lambda / \pi)$ as $\phi \sim \pi$ (Fano mode), respectively. Thus the distance $\mathrm{r}$ is $1.5 \sim 10 \mu \mathrm{m}$ as $\mathcal{\lambda}$ is $500 \mathrm{~nm}$ and $\left|k_{2} / k\right| \sim 10$, sufficiently short to a side length of a conventional LED chip.

Namely, not only an OLED but also a conventional LED may have the SW excitation loss within. 
In this research, our model is corresponding to a planer-type light-emitting device eliminating thin film layers. These film layers have a potential inducing a mode coupling between a surface EM wave and a waveguide mode, by which the SPPs can be controlled and the "surface plasmon loss" diminished ${ }^{1920)}$.

\section{Acknowledgement}

The author would like to thank Nobutaka TANIGAKI for his giving an opportunity of this research, also to thank Kensuke MURAI for his discussion mainly on SPPs.

\section{References}

(1) Reineke, S., Lindner, F., Schwartz, G., Seidler, N., Walzer, K., Lussen, B., and Leo, K.: White organic light-emitting diodes with fluorescent tube efficiency, Nature, Vol. 459, pp. 234-238 (2009).

(2) Lin, C., Cho, T., Chang, C., and Wu, C.: Enhancing light outcoupling of organic light-emitting devices by locating emitters around the second antinode of the reflective metal electrode, Apple. Phys. Lett., Vol. 88, 081114 (2006).

(3) Sullivan, K. G., and Hall, D. G.: Enhancement and inhibition of electromagnetic radiation in plane-layered media. I. plane-wave spectrum approach to modeling classical effects, J. Opt. Soc. Am., Vol. B14, pp. 1149-1166 (1997).

(4) Zenneck, J.: Ueber die Fortpflanzung ebener electromagnetisher Wellen laengs einer ebenen Leiterflache und ihre Beziehung zur drahtlosen Telegraphie, Ann. Phys., Vol. 23, pp. 847-866 (1907).

(5) Sommerfeld, A.: Ueber die Ausbreitung der Wellen in der drahtlosen Telegraphie, Ann. Phys., Vol. 28, pp. 665-736 (1909).

(6) Wait, J. R.: The ancient and modern history of EM ground-Wave Propagation, IEEE Antenna \& Propagat. Mag. Vol. 40, No. 5, pp. 7-24 (1998).

(7) Burstein, E., Chen, W. P., Chen, Y. J., and Hartstein, A.: Surface polaritons-propagating electromagnetic modes at interfaces, J. Vac. Sci. Tech., Vol. 11, pp. 1004-1019 (1974).

(8) Weber, W. H. and Eagan, C. F.: Energy transfer from an excited dye molecule to the surface plasmons of an adjacent metal", Opt. Lett., Vol. 4, pp. 236-238 (1979).

(9) Kawata, S.: Plasmons: Future outlook, J. J. Appl. Phys., Vol. 52, 010001 (2013).

(10) Ashcroft, N. W. and Mermin, N. D.: Solid state physics, Saunders, Chap. 1, Problem 5 (1976).

(11) Yeh, P.: Optical waves in layered media, Willey, Chap. 11, Sec. 4 (1988).

(12) Norton, K. A.: The propagation of radio waves over the surface of the earth and in the upper atmosphere, Proc. IRE, Vol. 24, No. 10, pp. 1367-1387 (1936); Ibid., Vol. 25, No. 9, pp. 1203-1236 (1937).

(13) Nomura, Y.: Electromagnetic-wave propagation over a plane, homogeneous earth's surface, J. IECE, Vol. 37, No. 3, pp. 160-165 (1954) in Japanese.

(14) Van der Pol, V. B. and Nissen, K. F.: Ueber die Raumwellen von einem vertikalen Dipolsender auf ebener Erde, Ann. Phys., Vol. 10, pp. 485-510 (1931).

(15) Sommerfeld, A.: Partielle Differentialgleichungen der Physik, Vorlesunge ueber Theoretische Physik VI, ver. 6, F. Sauter ed., Sec. 32 (1957); translated in Japanese by H. Masuda (1969).

(16) Norton, K. A.: The physical reality of space and surface waves in the radiation field of radio antennas, Proc. IRE, Vol. 25, No. 9, pp. 1192-1202 (1937).

(17) Barlow, H. M. and Cullen, A. L.: Surface waves, Proc. IEE, Part III, Vol. 100, pp. 329-341 (1953).

(18) Petrillo, L., Jangel, F., Darces, M., Montmagnon, J.-L., and Helier, M.: Negative permittivity media able to propagate a surface wave, Progress in Electromagn. Res., Vol. 115, pp. 1-10 (2011).

(19) Mikami, A.: Optical design of enhanced light extraction efficiency in organic light emitting device with an optically controlled surface plasmon coupling, J. Light \&Vis. Env., Vol. 37, No. 2 \& 3, pp. $62-65$ (2013).

(20) Ishido, Y. and Tanigaki, N.: The growth of out-coupling from a large thin-film electroluminescence by a guided mode transformation, The Papers of Technical Meeting, IEE Japan, EMT-14-069 (2014) in Japanese.

(21) In our precise research, however, this is not so correct as the $\pi / 2-\theta$ may be equated to the Brewster angle in analytic continuation with respect to a complex permittivity of a grounded medium: That may bring some suggestions to the oblique incident beam excitation in an apparatus for applying SPPs; for example, in the following and reference (8): Gryczynski, I., Malicka, J., Gryczynski, Z., and Lakowicz, J. R.: Surface Plasmon-Coupled Emission with Gold Films, J. Phys. Chem. B, Vol. 108, 12568-12574 (2004).

(22) The original definition is $0<\phi<\pi / 2$, following expanded with no remarking; see the following part of Appendix.

\section{Appendix. A steepest descent method for the field integrals and the $\mathrm{SW}$ poles}

Here is a review of reference (13) in Japanese, based on Van der Pol and Nissen's (14) in Deutch. In this section, the time factor exp $(\mathrm{j} \omega t)$ has been consistently omitted. Returning to the start point, let us adopt the Herzian vector of total fields represented by complex integrals with respect to a complex wave number $\mathcal{\lambda}$, as: 


$$
\Pi_{z}=2 \exp (-\mathrm{j} k R) / R-S
$$

In (A1), the field integral $S$ is;

$$
\begin{aligned}
S= & k^{2} \int_{-\infty}^{\infty}\left(\lambda^{2}-k_{2}^{2}\right)^{1 / 2}\left(\lambda^{2}-k^{2}\right)^{-1 / 2} N(\lambda)^{-1} \\
& \times \mathrm{H}_{0}^{(2)}(\lambda r) \exp \left\{-\left(\lambda^{2}-k_{2}^{2}\right)^{1 / 2} z\right\} \mathcal{\lambda} \mathcal{\lambda}
\end{aligned}
$$

The coefficient "2" in Equation (A1) means the radiation from an image potential being included in. And Equation (A2) corresponds to the "lateral" component of the fields.

The poles of the field integral $S$ are equivalent to the zeroes of $N(\lambda)$, corresponding to a dispersion relation of the SW:

$$
N(\lambda)=k^{2}\left(\lambda^{2}-k_{2}^{2}\right)^{1 / 2}+k_{2}^{2}\left(\lambda^{2}-k^{2}\right)^{1 / 2}=0
$$

Thus the pole $\lambda_{\mathrm{p}}$ is seen in the form;

$$
\lambda_{\mathrm{p}}= \pm k k_{2}\left(k^{2}+k_{2}^{2}\right)^{-1 / 2}
$$

$k$ and $k_{2}$ are wave numbers of a free space and a medium interfacing to it, defined as follows, respectively.

$$
\begin{array}{ll}
k_{2}^{2}=\left|k_{2}^{2}\right| \exp (-\mathrm{j} \phi) & \left(0<\phi<\pi^{22)}\right) \\
k^{2}=\left|k^{2}\right| \exp (-\mathrm{j} \varepsilon) \quad(\varepsilon \sim 0)
\end{array}
$$

It is remarked that $k / k_{2}$ should correspond to the notation $u$ in Equation (1) in the text.

For conveniences' sake, we change the integral variant as;

$$
\lambda=k \sin a
$$

Thus, Equation (A2) is modified as;

$$
\begin{aligned}
\mathrm{S}= & -\mathrm{j} k^{3} \pi^{-1} \int_{L} \mathrm{~d} \zeta \int_{C}\left(k^{2} \sin ^{2} a-k_{2}^{2}\right)^{1 / 2} \sin a N(\alpha)^{-1} \\
& \times \exp \{-\mathrm{j} k R(\cos \theta \sin a \sin \zeta+\sin \theta \cos \alpha)\} \mathrm{d} \alpha
\end{aligned}
$$

In Equation (A8), it is remarked;

$$
\sin \theta=z / R
$$

As to the integral paths $L$ and $C$, we adopt steepest descent paths (SDP), respectively. It corresponds to the case that the distance between a dipole and an observation point much longer than a free space wavelength.

The procedure of the integration using SDP as follows:

First, we decide saddle points in $L$ and $C$, with the derivation of the phase term of an exponential function in the integrand of Equation (A8) with respect to $\zeta$ and $a$, respectively:

$-\mathrm{j} k R \cos \theta \sin \alpha \cos \zeta=0$

$-\mathrm{j} k R(\cos \theta \cos a \sin \zeta-\sin \theta \sin \alpha)=0$

Thus, the saddle points are respectively as;

$$
\zeta_{0}=\pi / 2
$$

$$
a_{0}=\pi / 2-\theta
$$

Therefore, the field integral $S$ is;

$$
\begin{aligned}
S= & k^{3} \pi^{-1} \exp (-\mathrm{j} k R) \int_{-\infty}^{\infty} \exp \left\{-(1 / 2) k R \cos ^{2} \theta s_{2}^{2}\right\} \mathrm{d} s_{2} \\
& \times \int_{-\infty}^{\infty} \mathrm{j} k_{2} \sin a_{0} N(a)^{-1} \exp \left\{-(1 / 2) k R s_{1}^{2}\right\} \mathrm{d} s_{1} \\
& +K \text { (a Residue, if there is.) }
\end{aligned}
$$

In Equation (A13), $s_{1}$ and $s_{2}$ are defined as the followings, remarked to be both real, respectively:

$$
\zeta-\zeta_{0}=s_{2} \exp (\mathrm{j} \pi / 4), \alpha-a_{0}=s_{1} \exp (\mathrm{j} \pi / 4)
$$

On the other hand, as the pole $\lambda_{\mathrm{p}}$ transforms to the one in $a$ plane by Equation (A7);

$$
\begin{aligned}
& \sin a_{\mathrm{p}}=k\left(k^{2}+k_{2}^{2}\right)^{-1 / 2} \\
& \cos a_{\mathrm{p}}=-k_{2}\left(k^{2}+k_{2}^{2}\right)^{-1 / 2}
\end{aligned}
$$

Let us take at a glance of the physical meaning of the pole. If the phase $\phi$, which is equivalent to the loss angle of a medium interfacing to the free space, approaches to zero, the limiting value of $a_{\mathrm{p}}$ is known to be a Brewster angle as already mentioned. Hence, $a_{\mathrm{p}}$ is often called a complex Brewster angle ${ }^{17}$, which represents the gradient of phase planes of a Zenneck-Sommerfeld mode, as mentioned in the text.

If the absolute amount of $k / k_{2}$ is much less than 1 ,

$$
a_{\mathrm{p}}=\pi / 2+k / k_{2}
$$

As seen in Equations (A13) and (A16), $a_{\mathrm{p}}$ is located in the neighborhood of $a_{0}$. Therefore, we should adopt Taylor's expansion around $a_{0}$ to evaluate the dispersion relation function $N(\alpha)$ :

$$
\begin{aligned}
N(\alpha) & =N\left(a_{0}\right)+\{\partial N(\alpha) / \partial \alpha\}_{a=a_{0}}\left(a_{0}-\alpha\right) \\
& =-\mathrm{j} k k_{2}^{2} \exp (\mathrm{j} \pi / 4)\left(\mathrm{s}_{1}-T\right)
\end{aligned}
$$

In Equation (A17), the following $T$ is important;

$$
T=\left(\sin \theta+k / k_{2}\right) \exp (-\mathrm{j} \pi / 4)
$$

Note that, as Equation (A10), if $\sin \theta=z \ll R$, then $\sin$ $\theta \ll 1$, thus;

$$
\theta \sim z / R
$$

After all, the field integral $S$ is;

$$
\begin{aligned}
S= & -k_{2} k^{3} \pi^{-1 / 2} \exp (-\mathrm{j} k R)\left\{(1 / 2) k R \cos ^{2} \theta\right\}^{-1 / 2} \cos \theta \\
& \times \int_{-\infty}^{\infty}\left\{k k_{2}^{2} \exp (\mathrm{j} \pi / 4)\left(s_{1}-T\right)\right\}^{-1} \exp \left\{-(1 / 2) k R s_{1}^{2}\right\} \mathrm{d} s_{1} \\
& +K
\end{aligned}
$$

It is remarked that the integral in Equation (A20) yields Cauchy's Principal if and only if $T$ is a pure real.

Furthermore, the residue in Equation (A13), or (A20), $K$ is approximated, as $\sin a_{\mathrm{p}} \sim 1, r \sim R$, and $\sin \left(a_{\mathrm{p}}+\theta\right)$ $\sim 1-\mathrm{j} T^{2} / 2$;

$$
K \sim 2 \pi\left(k / k_{2}\right)(2 k \pi / R)^{1 / 2} \exp \left\{-\mathrm{j}(k R+\pi / 4)-(1 / 2) k R T^{2}\right\}
$$

In general, poles in the integral representation for 
EM field, or zeros of the dispersion relation, correspond to waveguide modes of the field, and their residues represent Fourier components of the modes.

A surface wave at the interface excited by a vertical electric dipole however, shows somewhat different aspects. According to reference (13), if the pole is right hand of the SDP, it means that the pole is outward of the domain of integration, thus there is no influence of the residue of the pole. On the other hand, if the pole is left hand of the SDP, the situation of the pole is improper $^{6)}$, of which a residue ought to be cancelled by a piece of integral term along the SDP. Wait mentioned in reference (6) that a spatial causality decides whether the pole is improper through the selection of the Riemann's sheet of a square root function.

At any rate, the residue seen in Equation (A20), is equal to the term of the Zenneck's surface wave, thus, as $\left|k / k_{2}\right| \ll 1$, the numerical distance $\rho$ shown in Equa- tion (3) is corresponding to:

$(1 / 2) k R T^{2}$

Using equation (A22) as $z \rightarrow 0$, Wait showed that the argument of a numerical distance ranges from $-\pi / 2$ to 0 as that of an inverse refractivity from 0 to $\pi / 4^{6)}$.

In our case, the permittivity $\varepsilon_{2}$, whose argument is augmented from 0 to $-\pi$, is concerned with the refractivity through the square root as seen in the definition of $u$ in Equation (1).

As a result of reference (13), with the adequate selection of a Riemann's sheet of the square root accounting a spatial causality for a free space wave number $\mathrm{k}$ as $\mathrm{k} \rightarrow \mathrm{k}-\mathrm{j} \kappa(0<\kappa \ll 1)$, that argument is suggested to range also from 0 to $-\pi / 2$ as $u$ ranges from $\pi / 4$ to $\pi / 2$. It is most noted that the alternative selection of a Riemann's sheet had led to a little barren controversies ${ }^{6)}$ nevertheless, a similar report has been seen still now ${ }^{18)}$. 